\begin{tabular}{|c|c|}
\hline Title & Large magnetocapacitance effect in magnetic tunnel junctions based on Debye Frohlich model \\
\hline Author(s) & Kaiju, Hideo; Takei, Masashi; Misawa, Takahiro; Nagahama, Taro; Nishii, Junji; Xiao, Gang \\
\hline Citation & $\begin{array}{l}\text { A pplied physics letters, 107(13), 132405-1-132405-5 } \\
\text { https://doi.org/10.1063/1.4932093 }\end{array}$ \\
\hline Issue Date & $2015-09-28$ \\
\hline Doc URL & http:/hdl.handle.net/2115/60214 \\
\hline Rights & $\begin{array}{l}\text { Copyright } 2015 \text { A merican Institute of Phy sics. This article may be downloaded for personal use only. A ny other use } \\
\text { requires prior permission of the author and the A merican Institute of Physics. The following article appeared in A ppl. } \\
\text { Phys. Lett. 107, 132405, 2015, and may be found at http://dx.doi.org/10.1063/1.4932093 }\end{array}$ \\
\hline Type & article \\
\hline File Information & 1.4932093.pdf \\
\hline
\end{tabular}

Instructions for use 


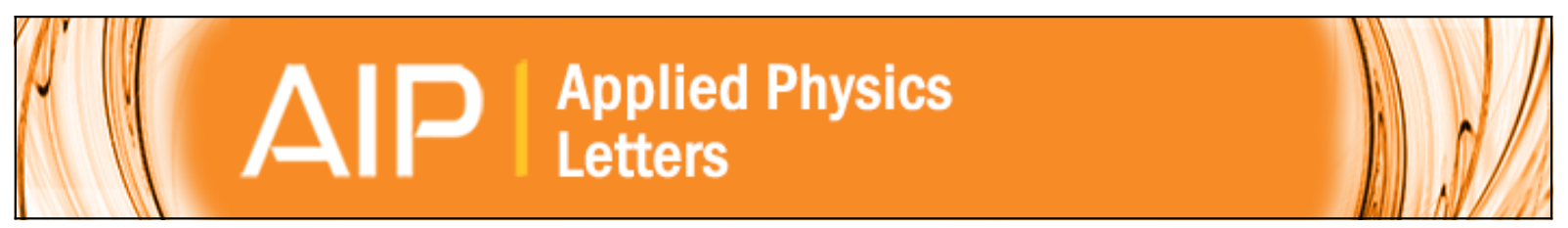

\section{Large magnetocapacitance effect in magnetic tunnel junctions based on Debye-}

\section{Fröhlich model}

Hideo Kaiju, Masashi Takei, Takahiro Misawa, Taro Nagahama, Junji Nishii, and Gang Xiao

Citation: Applied Physics Letters 107, 132405 (2015); doi: 10.1063/1.4932093

View online: http://dx.doi.org/10.1063/1.4932093

View Table of Contents: http://scitation.aip.org/content/aip/journal/apl/107/13?ver=pdfcov

Published by the AIP Publishing

\section{Articles you may be interested in}

Sub-Poissonian shot noise in $\mathrm{CoFeB} / \mathrm{MgO} / \mathrm{CoFeB}-$ based magnetic tunneling junctions

Appl. Phys. Lett. 98, 202103 (2011); 10.1063/1.3590921

Observation of full shot noise in $\mathrm{CoFeB} / \mathrm{MgO} / \mathrm{CoFeB}-$ based magnetic tunneling junctions

Appl. Phys. Lett. 96, 252504 (2010); 10.1063/1.3456548

Sign of tunneling magnetoresistance in $\mathrm{Cr} O 2$-based magnetic tunnel junctions

Appl. Phys. Lett. 91, 252506 (2007); 10.1063/1.2825475

Frequency-dependent magnetoresistance and magnetocapacitance properties of magnetic tunnel junctions with MgO tunnel barrier

Appl. Phys. Lett. 90, 142105 (2007); 10.1063/1.2719032

Spin-transfer switching in MgO-based magnetic tunnel junctions (invited)

J. Appl. Phys. 99, 08G510 (2006); 10.1063/1.2165169

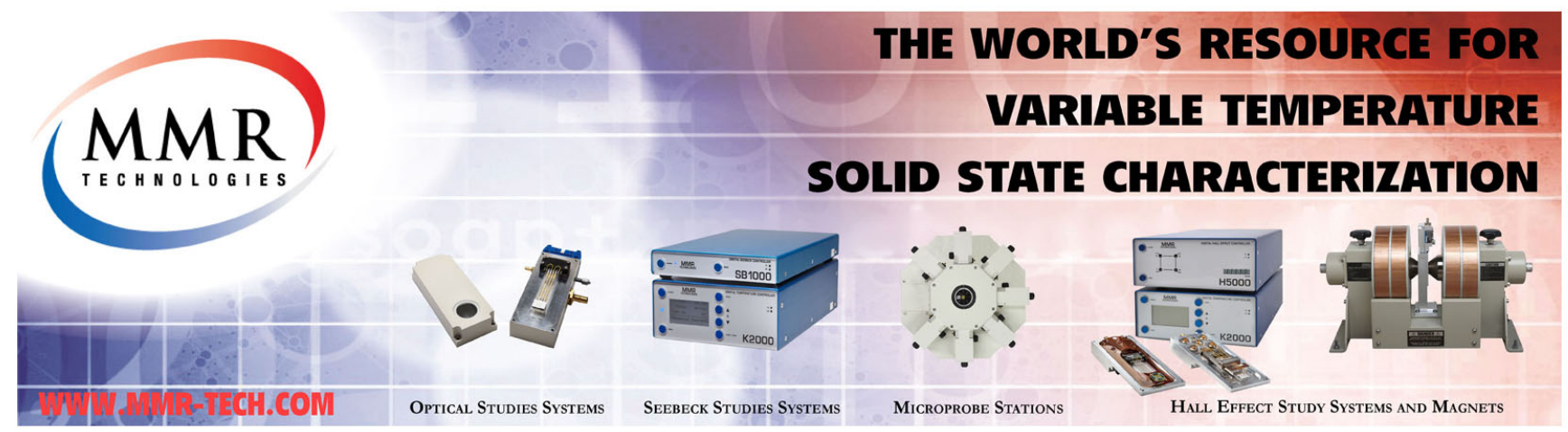




\title{
Large magnetocapacitance effect in magnetic tunnel junctions based on Debye-Fröhlich model
}

\author{
Hideo Kaiju, ${ }^{1, a)}$ Masashi Takei, ${ }^{1}$ Takahiro Misawa, ${ }^{1}$ Taro Nagahama, ${ }^{2}$ Junji Nishii, ${ }^{1}$ \\ and Gang Xiao ${ }^{3}$ \\ ${ }^{1}$ Research Institute for Electronic Science, Hokkaido University, Sapporo, Hokkaido 001-0020, Japan \\ ${ }^{2}$ School of Engineering, Hokkaido University, Sapporo, Hokkaido 060-8628, Japan \\ ${ }^{3}$ Department of Physics, Brown University, Providence, Rhode Island 02912, USA
}

(Received 15 July 2015; accepted 19 September 2015; published online 29 September 2015)

\begin{abstract}
The frequency dependence of tunneling magnetocapacitance (TMC) in magnetic tunnel junctions (MTJs) is investigated theoretically and experimentally. According to the calculation based on Debye-Fröhlich model combined with Julliere formula, the TMC ratio strongly depends on the frequency and it has the maximum peak at a specific frequency. The calculated frequency dependence of TMC is in good agreement with the experimental results obtained in MgO-based MTJs with a tunneling magnetoresistance (TMR) ratio of $108 \%$, which exhibit a large TMC ratio of $155 \%$ at room temperature. This calculation also predicts that the TMC ratio can be as large as about $1000 \%$ for a spin polarization of $87 \%$, while the TMR ratio is $623 \%$ for the same spin polarization. These theoretical and experimental findings provide a deeper understanding on $\mathrm{AC}$ spin-dependent transport in the MTJs and will open up wider opportunities for device applications, such as highly sensitive magnetic sensors and impedance-tunable devices. (C) 2015 AIP Publishing LLC.

[http://dx.doi.org/10.1063/1.4932093]
\end{abstract}

Magnetic tunnel junctions (MTJs) have played an important role for fundamental understanding of spindependent phenomena and applications to read heads in hard disk drives, highly sensitive magnetic sensors, and magnetic random access memory. ${ }^{1-4}$ One of the most successful developments in the MTJs is the band-calculation prediction ${ }^{5,6}$ and experimental demonstration ${ }^{3,4}$ of large tunneling magnetoresistance (TMR) based on coherent tunneling process. At present, the TMR ratio of the MTJs with $\mathrm{MgO}$ tunnel barriers exhibits as large as $600 \%^{7}$ at room temperature and over $2000 \%{ }^{8}$ at low temperature. On the other hand, AC transport has also been studied in the MTJs due to their fascinating spin phenomena, such as frequency-dependent spin transport and spin capacitance, and applications to high-speed storage devices and high-frequency elements, both theoretically and experimentally. ${ }^{9-17}$ One of the most attractive AC spin transport properties is the tunneling magnetocapacitance (TMC), which has been observed in $\mathrm{Al}_{2} \mathrm{O}_{3^{-}}$or $\mathrm{MgO}$-based MTJs. ${ }^{11-13,16,17}$ In TMC effect, the capacitance is high for the parallel $(\mathrm{P})$ configuration of the magnetization vectors in both ferromagnetic layers adjacent to the barrier and it is low for the antiparallel (AP) configuration. This phenomenon is explained by the spin-injection accumulation model. ${ }^{16}$ Foe example, a large amount of charge accumulates in the interface between the ferromagnetic layer and insulator for P configuration due to the high conductance, meanwhile a small amount of charge accumulates for AP configuration due to the low conductance. Based on this picture, the TMC is found to be strongly correlated with the TMR. However, the reported TMC ratio is about $50 \%$, which is much smaller than what is expected from the above-mentioned TMR ratio. ${ }^{13}$ Additionally, the TMC depends on the frequency, ${ }^{11-13}$

${ }^{\text {a)} E-m a i l: ~ k a i j u @ e s . h o k u d a i . a c . j p ~}$ and in some cases, the TMC is reported to be negligible. ${ }^{18}$ These observations suggest that a further theoretical consideration and a more detailed experimental investigation are required to fully understand the TMC effect.

Recently, the magneto-dielectric effect has been observed in $\mathrm{FeCo}-\mathrm{MgF}$ nanogranular films at room temperature, which consist of nanometer-sized FeCo magnetic particles dispersed in an $\mathrm{MgF}$ dielectic matrix. ${ }^{19,20}$ Since the dielectric constant, obtained from capacitance measurement, is dependent on the magnetic field and the electron transport is dominated by spin-dependent tunneling, this magnetodielectric phenomenon is a particular kind of TMC effect. Although the TMC ratio, corresponding to the change in the dielectric constant, is small at 3.0\%-6.2\% in this system, the magnetoelectric response is well explained by the theoretical calculation based on Debye-Fröhlich model. ${ }^{19}$ This model can also be applied to the TMC effect in the MTJs, because it is valid for the calculation of dynamic dielectric constant in a wide variety of insulating solid and liquid systems. ${ }^{21-24}$

In this letter, we present the frequency dependence of TMC in the MTJs theoretically and experimentally. According to our calculation based on Debye-Fröhlich model combined with Julliere formula, the TMC ratio strongly depends on frequency with a maximum peak at a specific frequency. The calculated frequency dependence of TMC is in good agreement with the experimental results obtained in MgO-based MTJs with a TMR ratio of $108 \%$, which exhibit a large TMC ratio of $155 \%$ at room temperature. This calculation also predicts that the TMC ratio can reach $1000 \%$ in the MTJs with a corresponding TMR ratio of $623 \%$.

The calculation of the frequency characteristics of TMC is performed using the above-mentioned Debye-Fröhlich model combined with Julliere formula. The Debye-Fröhlich model is a useful tool for the calculation of dynamic 
dielectric constant in a variety of materials, ranging from paramagnetic and dielectric media ( $\mathrm{Fe}-, \mathrm{Cr}$-, and $\mathrm{V}$-alum), ordinary metals $(\mathrm{Al}, \mathrm{W}$, and $\mathrm{Pb}$ ) to liquids (alcohols, glycols, and benzene). ${ }^{21-24}$ Based on the model, ${ }^{25}$ the complex dielectric constant $\varepsilon^{*}$ can be generally represented by

$$
\varepsilon^{*}=\varepsilon_{\infty}+\frac{\varepsilon_{0}-\varepsilon_{\infty}}{1+(i \omega \tau)^{\beta}},
$$

where $\varepsilon_{\infty}$ and $\varepsilon_{0}$ are the high-frequency and static dielectric constants, $\omega$ is the angular frequency, and $\tau$ is the relaxation time. Here, it should be noted that the exponent $\beta$, indicating the distribution of relaxation time, is a very important parameter $(0<\beta \leq 1)$. This is because involving the exponent $\beta$ in Eq. (1) allowed us to explain the experimental results in a considerable number of dielectrics. ${ }^{24}$ Since the MTJ includes the dielectrics, the Debye-Fröhlich model can be valid for the calculation of its dielectric constant, whose real part is proportional to the capacitance. Thus, the capacitance $C_{\mathrm{P}(\mathrm{AP})}$ of the $\mathrm{P}(\mathrm{AP})$ configuration in the MTJs can be expressed by

$$
C_{P(A P)}(f)=\operatorname{Re}\left[C_{\infty}+\frac{C_{0}-C_{\infty}}{1+\left(i 2 \pi f \tau_{P(A P)}\right)^{\beta}}\right]
$$

where $C_{\infty}$ and $C_{0}$ are the high-frequency and static capacitances, $f$ is the frequency, and $\tau_{\mathrm{P}(\mathrm{AP})}$ is the relaxation time in $\mathrm{P}(\mathrm{AP})$ configuration. From Julliere formula, ${ }^{26}$ the relation between $\tau_{\mathrm{P}}$ and $\tau_{\mathrm{AP}}$ is given by

$$
\tau_{A P}=\frac{1+P^{2}}{1-P^{2}} \tau_{P}
$$

where $P$ is the spin polarization of the ferromagnetic layer. Therefore, as the TMC ratio is defined by

$$
\mathrm{TMC} \text { ratio }=\frac{C_{P}(f)-C_{A P}(f)}{C_{A P}(f)},
$$

substituting $C_{\infty}, C_{0}, \beta, \tau_{\mathrm{P}}$, and $P$ in Eqs. (2) and (3) gives the frequency characteristics of the TMC ratio. The TMC effect is often explained by Maxwell-Wagner model, which is expressed by $R C$ parallel equivalent circuit. ${ }^{13,16}$ The capacitance in this conventional model is independent of the frequency. In contrast, the capacitance in our model depends on the frequency in accordance with the expression of Eq. (2), which is based on Debye-Fröhlich model. The equivalent circuit is shown in Fig. 1(a). Namely, our model is a special case of Maxwell-Wagner model, i.e., the frequency dependence of the dielectric constant based on Debye-Fröhlich model is taken in Maxwell-Wagner model. The physical picture is shown in Fig. 1(b), which represents a schematic of the electric potential profile of the MTJ (two magnetic layers separated by the thin insulator) in an AC electric field $E$. The electric carriers (electrons or holes) are tunneling through the barrier in accordance with the AC field. Then, the electric dipole is formed by electrons and holes and oscillates under the AC field. The dynamic behavior of the dipole obeys the Debye-Fröhlich model; in the low frequency region, the dipole follows the change of the electric field due to the low speed of $\mathrm{AC}$ field, and in the high frequency region, the dipole does not follow the change of the electric field. Since (a)

(b) Electric potential

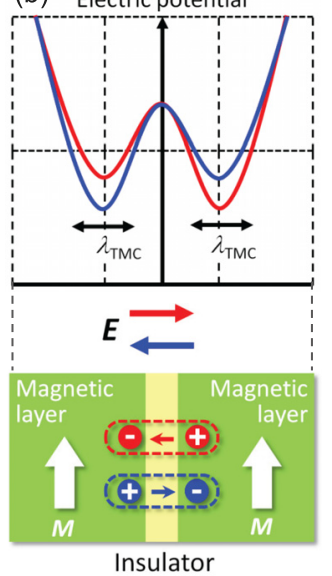

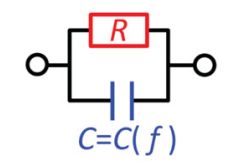

(c)

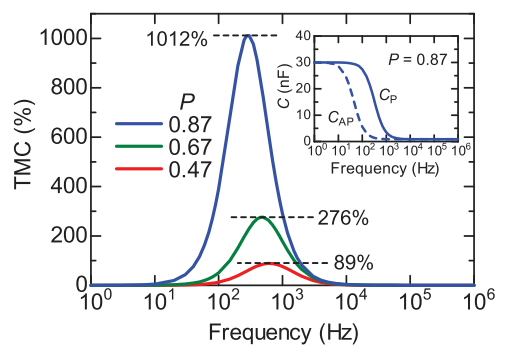

(d)

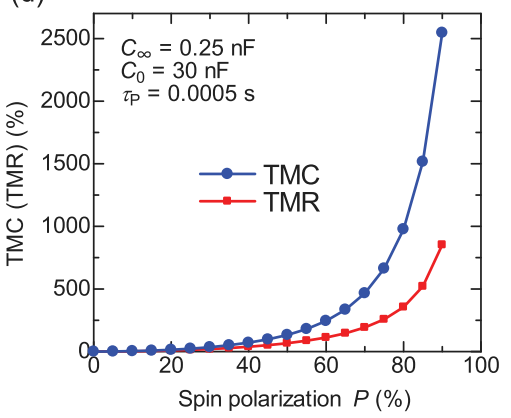

FIG. 1. (a) Equivalent circuit of MTJs based on Debye-Fröhlich model and (b) a schematic of the electric potential profile of MTJs in an AC electric field $E$. (c) Frequency dependence of TMC ratio obtained from the calculation based on Debye-Fröhlich model combined with Julliere formula for $C_{\infty}=0.85 \mathrm{nF}, C_{0}=30 \mathrm{nF}, \beta=1.0$, and $\tau_{\mathrm{P}}=5.0 \times 10^{-4} \mathrm{~s}$ in Eqs. (2)-(4). The inset shows the frequency dependence of $C_{\mathrm{P}}$ and $C_{\mathrm{AP}}$ for $P=87 \%$. (d) Spin polarization dependence of TMC and TMR ratios for $C_{\infty}=0.25 \mathrm{nF}$, $C_{0}=30 \mathrm{nF}$, and $\tau_{\mathrm{P}}=5.0 \times 10^{-4} \mathrm{~s}$.

the relaxation time $\tau_{\mathrm{P}(\mathrm{AP})}$ is spin dependent, the frequency characteristics of the capacitance $C_{\mathrm{P}(\mathrm{AP})}$ are shifted in accordance with the magnetization state of ferromagnetic layers. In this shift region, a large TMC effect can be observed.

The calculated frequency dependence of TMC ratio in varying $P$ is shown in Fig. 1(c). Here, we set $C_{\infty}=0.85 \mathrm{nF}$, $C_{0}=30 \mathrm{nF}, \beta=1.0$, and $\tau_{\mathrm{P}}=5.0 \times 10^{-4} \mathrm{~s}$. The $\tau_{\mathrm{AP}}$ is calculated using Eq. (3). The TMC ratio strongly depends on the frequency and has the maximum peak. The maximum value of TMC ratio increases from $89 \%$ to $1012 \%$ with increasing $P$ from 0.47 to 0.87 . The TMC ratios of $89 \%, 276 \%$, and $1012 \%$ are larger than the TMR ratios of $57 \%, 163 \%$, and $623 \%$, which are calculated from Julliere formula, given by $\mathrm{TMR}=2 P^{2} /\left(1-P^{2}\right)$, using $P=0.47,0.67$, and 0.87 , respectively. The inset shows the frequency dependence of $C_{\mathrm{P}}$ and $C_{\mathrm{AP}}$ for $P=87 \%$. A significant difference between $C_{\mathrm{P}}$ and $C_{\mathrm{AP}}$ can be seen in the frequency region from $10 \mathrm{~Hz}$ to $1 \mathrm{kHz}$ and the TMC ratio shows a large value in this region. Because the frequency, exhibiting the maximum TMC ratio, is equal to the one, satisfying $d(\mathrm{TMC}) / d f=0$, the frequency $f_{\text {peak }}$ can be obtained as

$$
f_{\text {peak }}=\frac{1}{2 \pi \tau_{P}}\left(\frac{C_{0}}{C_{\infty}}\right)^{\frac{1}{4}} \sqrt{\frac{1-P^{2}}{1+P^{2}}},
$$

where $\beta$ is set to 1.0. Substituting $f=f_{\text {peak }}$ in Eqs. (2) and (4) gives the maximum TMC ratio. The $P$ dependence of the maximum TMC and TMR ratios is shown in Fig. 1(d). Here, we set $C_{\infty}=0.25 \mathrm{nF}, C_{0}=30 \mathrm{nF}$, and $\tau_{\mathrm{P}}=5.0 \times 10^{-4} \mathrm{~s}$. It is evident that the TMC ratio increases with increasing $P$ and it exceeds $1000 \%$ at $P>80 \%$. Also, the TMC ratio is larger 
than the TMR ratio in the entire $P$ region. Typically, the TMC ratio shows as large as $1516 \%$ for $P=85 \%$, whereas the TMR ratio is $441 \%$ for the same spin polarization.

In order to verify the validity of the calculation, we investigate the frequency dependence of $\mathrm{TMC}$ in $\mathrm{MgO}$ based MTJs experimentally. The MgO-based MTJs, shown in Fig. 2(a), were fabricated on thermally oxidized silicon wafers using a high vacuum magnetron sputtering system. The base pressure is $2 \times 10^{-8}$ Torr. The antiferromagnetic solid is a 15-nm IrMn film which is used to pin the bottom ferromagnetic electrode. The top and bottom ferromagnetic electrodes are 3-nm $\mathrm{Co}_{40} \mathrm{Fe}_{40} \mathrm{~B}_{20}$ films, respectively, and the tunnel barrier is a 2-nm $\mathrm{MgO}$ film. All layers except for $\mathrm{MgO}$ were deposited by $\mathrm{DC}$ magnetron sputtering at a constant Ar pressure of 1.5 mTorr. The $\mathrm{MgO}$ layer was deposited by radio frequency (RF) magnetron sputtering at an $\mathrm{Ar}$ pressure of 1.1 mTorr. For patterning MTJs, a standard photolithography and ion-milling processes were used. The junction area is $1800 \mu \mathrm{m}^{2}$ in an elliptical shape. As a final step of the fabrication, a magnetic thermal annealing of MTJs was performed in a vacuum chamber $\left(\sim 1 \times 10^{-6}\right.$ Torr $)$ to define the pinning axis for the bottom magnetic electrode. A uniform magnetic field of $4.5 \mathrm{kOe}$ is applied in the plane of MTJs. Annealing occurs at $310^{\circ} \mathrm{C}$ for $4 \mathrm{~h}$ with natural cooling. The frequency characteristics of the TMR and TMC in MTJs were measured by an AC four-probe method using an Agilent Technologies 4284A LCR meter in the frequency range from $80 \mathrm{~Hz}$ to $1 \mathrm{MHz}$ under an applied magnetic field up to $1.4 \mathrm{kOe}$ at room temperature.

Typical TMR and TMC curves of an MTJ are shown in Figs. 2(b) and 2(c). The frequency is $200 \mathrm{~Hz}$ and the AC voltage is $0.26 \mathrm{mV}_{\mathrm{rms}}$. The black arrows represent the sweeping direction of the magnetic field. The equivalent circuit of the MTJ is modeled by the $R C$ parallel network as shown in the inset. Although various types of equivalent circuit models, such as the parallel-leaky capacitance model and the geometric and interface capacitance model, ${ }^{13,16,27}$ have been proposed for MTJs, the experimental results support the parallel network model, consisting of the DC resistance $R$ and the effective capacitance $C$, in any case. From Fig. 2(b), it can be seen that the ordinary TMR effect is confirmed. The TMR ratio, defined by $\left(R_{\mathrm{AP}}-R_{\mathrm{P}}\right) / R_{\mathrm{P}}$, is $108 \%$, which is identical to that measured by a DC four-probe method. In addition to this, it can be found that the TMC effect is also observed, i.e., the capacitance $C_{\mathrm{P}}$ is high for the $\mathrm{P}$ configuration and the capacitance $C_{\mathrm{AP}}$ is low for the $\mathrm{AP}$ configuration. The TMC ratio is $155 \%$, which is larger than the TMR ratio. The behavior of $C_{\mathrm{P}}>C_{\mathrm{AP}}$ in the TMC effect (a)

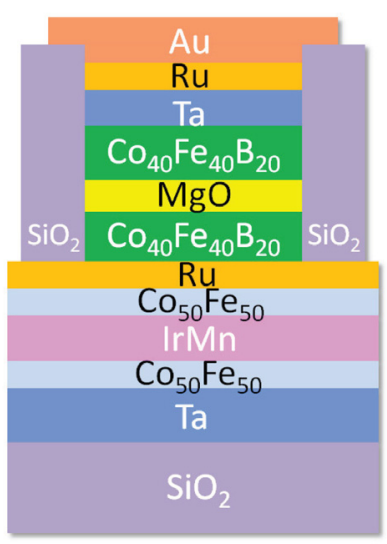

(b)

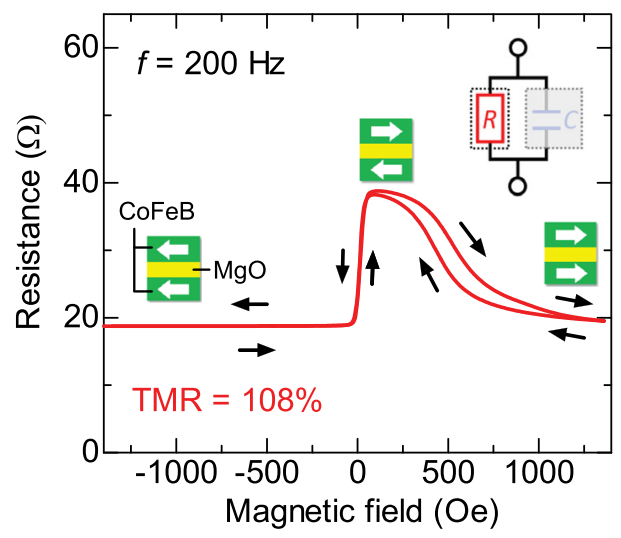

(c)

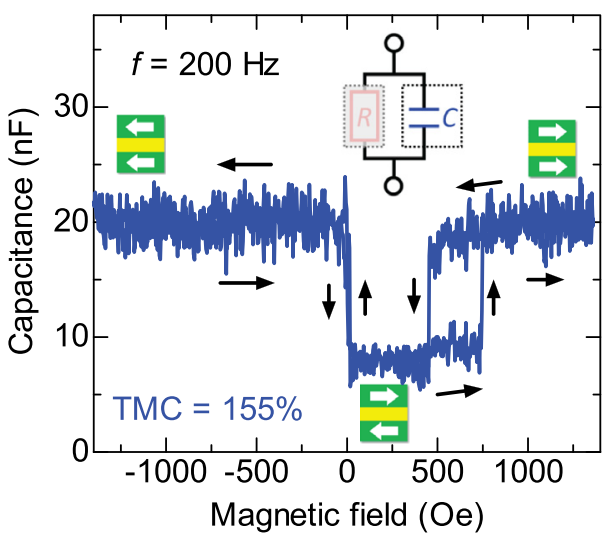

(d)

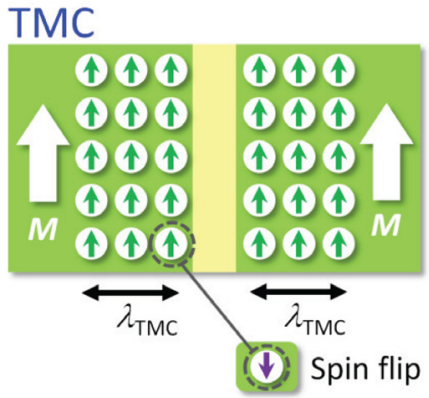

(e)

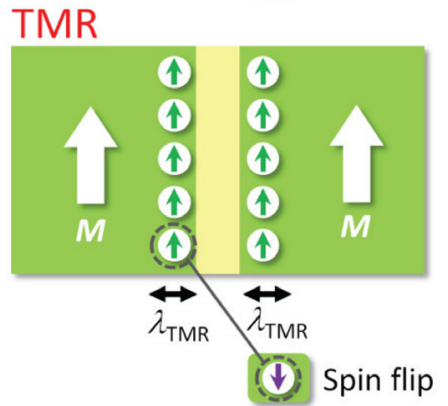

FIG. 2. (a) Schematic illustration of MgO-based MTJs. (b) TMR and (c) TMC curves of a MgO-based MTJ with a junction area of $1800 \mu \mathrm{m}^{2}$ at a frequency of $200 \mathrm{~Hz}$. Penetration length into the ferromagnetic layers of spin-dependent carriers contributing to (d) TMC and (e) TMR. 
can be well explained by putting $\tau_{\mathrm{AP}}>\tau_{\mathrm{P}}$, which is obvious from Eq. (3), into Eq. (2). Moreover, the observation of TMC > TMR is consistent with the calculation results shown in Fig. 1(d). Here, it should be noted that the TMC changes sharply at 500 and $750 \mathrm{Oe}$, whereas the TMR changes gradually around $500 \mathrm{Oe}$. The difference between TMC and TMR behaviors is attributed to the penetration length $\lambda$ into the ferromagnetic layers of spin-dependent carriers (electrons or holes) contributing to TMC and TMR. In TMC, as shown in Fig. 1(b), spin-dependent carriers with a certain width of $\lambda_{\text {TMC }}(\sim \mathrm{nm})$ contribute to AC transport. This means that the TMC is determined by spin polarization of carriers in the ferromagnetic layers near the interface between ferromagnetic and insulating layers, as shown in Fig. 2(d). Therefore, there is little influence on TMC even though the magnetization states in the interface are partly changed due to some intrinsic or extrinsic reasons, such as the insufficient magnetic pinning or the existence of interface roughness and impurity. Since spin-dependent carriers contributing to TMC is dominated by the entire magnetization states near the interface, the sharp change of TMC is observed. On the other hand, the TMR is determined by spin polarization of carriers in the ferromagnetic layers in the interface between ferromagnetic and insulating layers, i.e., the penetration length $\lambda_{\text {TMR }}$ is short, typically, an atomic order. Therefore, there is a large influence on the TMR even though the magnetization states in the interface are partly changed, as shown in Fig. 2(e). Since the TMR is sensitive to the magnetization states in the interface, the gradual change of TMR is observed.

To obtain a strong evidence that the Debye-Fröhlich model is valid for the calculation of TMC, the frequency dependence of TMR and TMC is investigated. The TMC curves in the frequency range from $80 \mathrm{~Hz}$ to $100 \mathrm{kHz}$ are shown in Fig. 3(a). The TMC ratio shows a maximum value of $155 \%$ at $200 \mathrm{~Hz}$ and it decreases down to $0 \%$ with increasing the frequency. The switching field, attributed to the magnetization reversal, is not changed over the entire frequency range. TMR curves (not shown here) exhibit the same behavior as that in Fig. 2(b), at any frequency.

The frequency dependence of TMR, TMC, and $C_{\mathrm{P}(\mathrm{AP})}$ is shown in Figs. 3(b) and 3(c). The calculation of TMC and $C_{\mathrm{P}(\mathrm{AP})}$ is performed by setting $C_{\infty}=0.95 \mathrm{nF}, C_{0}=84.55 \mathrm{nF}$, $\beta=0.95, \tau_{\mathrm{P}}=1.25 \times 10^{-3} \mathrm{~s}$, and $P=0.593$ in Eqs. (2)-(4). TMR is also calculated by using the same $P=0.593$ in Julliere formula. It can be seen that the calculation results of TMR, TMC, and $C_{\mathrm{P}(\mathrm{AP})}$ provide excellent fits to experimental data.

We note that this model can also account for experimental results reported previously. For example, a strong frequency dependence of TMC has been observed in $\mathrm{Al}_{2} \mathrm{O}_{3^{-}}$ based MTJs at room temperature. ${ }^{11}$ The TMC increases up from around $10-100 \mathrm{kHz}$ and it shows the maximum value of $8 \%$ at a frequency of $1 \mathrm{MHz}$. As shown in Fig. 4(a), the maximum peak of TMC is shifted to the high frequency region in short $\tau_{\mathrm{P}}$. Also, from Fig. 4(b), the frequency behavior in TMC is broadened with decreasing $\beta$. These calculation results indicate that the peak position and broadening in the frequency dependence of the TMC can be controlled by changing $\tau_{\mathrm{P}}$ and $\beta$, respectively. Since the maximum value of TMC depends on $P$ as shown in Fig. 1(d), we can easily (a)

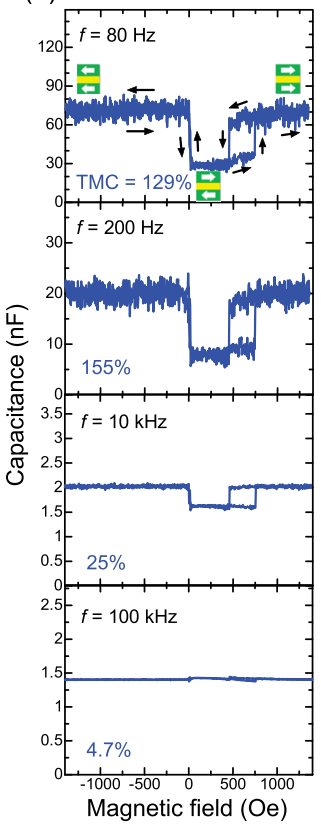

(b)

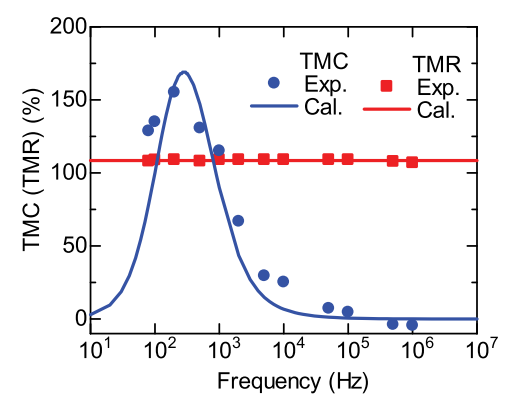

(c)

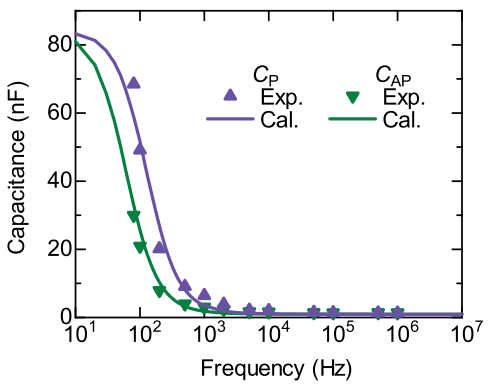

FIG. 3. (a) TMC curves of an MgO-based MTJ in the frequency range from $80 \mathrm{~Hz}$ to $100 \mathrm{kHz}$. Frequency dependence of (b) TMR and TMC ratios and (c) the capacitance $C_{\mathrm{P}(\mathrm{AP})}$ of the parallel (antiparallel) configuration of a MgO-based MTJ. The solid plots represent the experimental data and the solid lines represent the calculation results obtained from Debye-Fröhlich model; the parameters are set to $C_{\infty}=0.95 \mathrm{nF}, C_{0}=84.55 \mathrm{nF}, \beta=0.95$, $\tau_{\mathrm{P}}=1.25 \times 10^{-3} \mathrm{~s}$, and $P=0.593$, respectively, in Eqs. (2)-(4).

fit the experimental data with calculation curves. As a result of the fitting procedure, it is found that the experimental results are in good agreement with the calculation results obtained by assuming the following parameters: $C_{\infty}=5 \mathrm{nF}$, $C_{0}=455 \mathrm{nF}, \beta=0.97, \tau_{\mathrm{P}}=7 \times 10^{-7} \mathrm{~s}$, and $P=0.156$. In another case, it is reported that the TMC is lower than the TMR in $\mathrm{Al}_{2} \mathrm{O}_{3}$ - or MgO-based MTJs. ${ }^{13,28}$ Calculated TMC and TMR ratios as a function of $P$ are shown in Fig. 4(c). It is found that the TMC is lower than the TMR for a large $C_{\infty}$. Also, from Fig. 4(b), the maximum TMC (=99\%) is lower than the TMR $(=163 \%)$, which is calculated by Julliere formula, for a small $\beta$ of 0.75 . Thus, the experimental results of TMC $<$ TMR can be explained by substituting appropriate parameters of $C_{\infty}$ and $\beta$ for Debye-Fröhlich model. Finally, in one more example, the TMC is found to be quite low or
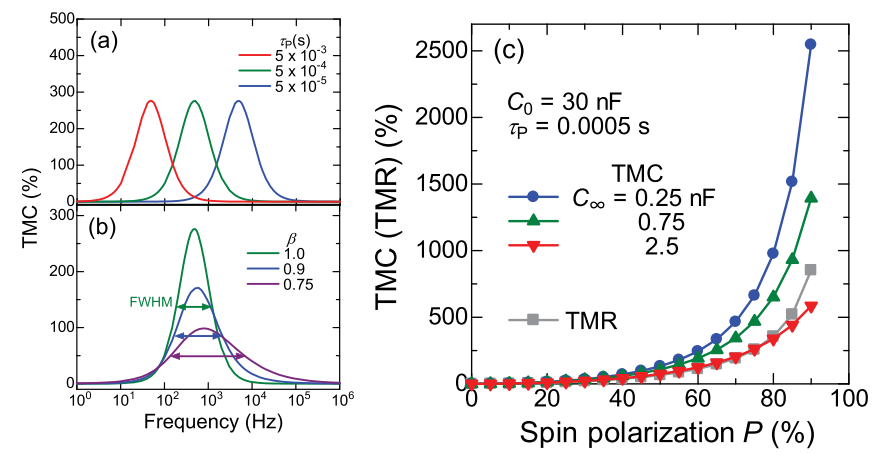

FIG. 4. Calculated frequency dependence of TMC ratios in varying (a) $\tau_{\mathrm{P}}$ for $\beta=1.0$ and (b) $\beta$ for $\tau_{\mathrm{P}}=0.0005 \mathrm{~s}$, respectively; $C_{\infty}=0.85 \mathrm{nF}, C_{0}=30$ $\mathrm{nF}$, and $P=0.67$. (c) Spin polarization dependence of TMR and TMC ratios for $C_{0}=30 \mathrm{nF}$ and $\tau_{\mathrm{P}}=5.0 \times 10^{-4} \mathrm{~s}$ in varying $C_{\infty}$. 
even absent in some cases. ${ }^{18,28}$ This can be easily understood from Fig. 4(a). Namely, the maximum peak of TMC is shifted to the high or low frequency region in short or long $\tau_{\mathrm{P}}$, in other words, a quite low TMC region is changed by $\tau_{\mathrm{P}}$. The TMC can be quite low or absent in certain frequency region. Thus, various experimental results reported previously can also be well explained by Debye-Fröhlich model combined with Julliere formula.

In summary, our detailed study on the TMC effect and earlier reports support the validity of our proposed TMC model, which predicts an extremely large TMC ratio of more than $1000 \%$ in MTJs with $P>80 \%$ at room temperature. These theoretical and experimental findings provide a deeper understanding on the AC spin transport in MTJs. The large TMC effect will offer wider opportunities for device applications, such as highly sensitive magnetic sensors and impedance-tunable devices. Electrical engineering of MTJ circuits must take the TMC effect into full consideration, particularly, in the frequency range where the TMC is most prominent.

This research was partially supported by a Grant-in-Aid for Scientific Research (B) from the Japan Society for the Promotion of Science (JSPS) and National Science Foundation through Grant No. DMR-1307056 at Brown University.

${ }^{1}$ T. Miyazaki and N. Tezuka, J. Magn. Magn. Mater. 139, L231 (1995).

${ }^{2}$ J. S. Moodera, L. R. Kinder, T. M. Wong, and R. Meservey, Phys. Rev. Lett. 74, 3273 (1995).

${ }^{3}$ S. Yuasa, T. Nagahama, A. Fukushima, Y. Suzuki, and K. Ando, Nat. Mater. 3, 868 (2004).

${ }^{4}$ S. S. P. Parkin, C. Kaiser, A. Panchula, P. M. Rice, B. Hughes, M. Samant, and S.-H. Yang, Nat. Mater. 3, 862 (2004).
${ }^{5}$ W. H. Butler, X.-G. Zhang, T. C. Schulthess, and J. M. MacLaren, Phys. Rev. B 63, 054416 (2001)

${ }^{6}$ J. Mathon and A. Umersky, Phys. Rev. B 63, 220403R (2001).

${ }^{7}$ S. Ikeda, J. Hayakawa, Y. Ashizawa, Y. M. Lee, K. Miura, H. Hasegawa, M. Tsunoda, F. Matsukura, and H. Ohno, Appl. Phys. Lett. 93, 082508 (2008).

${ }^{8}$ H.-X. Liu, T. Kawami, K. Moges, T. Uemura, M. Yamamoto, F. Shi, and P. M. Voyles, J. Phys. D: Appl. Phys. 48, 164001 (2015).

${ }^{9}$ S. Zhang, Phys. Rev. Lett. 83, 640 (1999).

${ }^{10}$ S. T. Chui and L. Hu, Appl. Phys. Lett. 80, 273 (2002).

${ }^{11}$ H. Kaiju, S. Fujita, T. Morozumi, and K. Shiiki, J. Appl. Phys. 91, 7430 (2002).

${ }^{12}$ W. C. Chien, C. K. Lo, L. C. Hsieh, Y. D. Yao, X. F. Han, Z. M. Zeng, T. Y. Peng, and P. Lin, Appl. Phys. Lett. 89, 202515 (2006).

${ }^{13}$ P. Padhan, P. LeClair, A. Gupta, K. Tsunekawa, and D. D. Djayaprawira, Appl. Phys. Lett. 90, 142105 (2007).

${ }^{14}$ K. M. Kuo, C. Y. Lin, C. T. Lin, G. Chern, C. T. Chao, L. Horng, J. C. Wu, T. Wu, C. Y. Huang, H. Ohyama, S. Isogami, M. Tsunoda, and M. Takahashi, Solid State Commun. 150, 1856 (2010).

${ }^{15}$ M.-F. Kuo, C.-M. Fu, X.-F. Han, C.-O. Chang, and C.-S. Chou, J. Appl. Phys. 109, 07C718 (2011).

${ }^{16}$ A. M. Sahadevan, K. Gopinadhan, C. S. Bhatia, and H. Yang, Appl. Phys. Lett. 101, 162404 (2012).

${ }^{17}$ M. Arikan, S. Ingvarsson, M. Carter, and G. Xiao, IEEE Trans. Magn. 49, 5469 (2013).

${ }^{18}$ S. Ingvarsson, M. Arikan, M. Carter, W. Shen, and G. Xiao, Appl. Phys. Lett. 96, 232506 (2010).

${ }^{19}$ N. Kobayashi, H. Masumoto, S. Takahashi, and S. Maekawa, Nat. Commun. 5, 4417 (2014).

${ }^{20}$ N. Kobayashi, T. Iwasa, K. Ishida, and H. Masumoto, J. Appl. Phys. 117, 014101 (2015).

${ }^{21}$ C. J. Gorter and R. de L. Kronig, Physica 3, 1009 (1936).

${ }^{22}$ H. B. G. Casimir and F. K. du Pre, Physica 5, 507 (1938).

${ }^{23}$ C. Zener, Phys. Rev. 53, 90 (1938).

${ }^{24}$ K. S. Cole and R. H. Cole, J. Chem. Phys. 9, 341 (1941).

${ }^{25} \mathrm{H}$. Fröhlich, Theory of Dielectrics: Dielectric Constant and Dielectric Loss (Clarendon, Oxford, 1958).

${ }^{26}$ M. Julliere, Phys. Lett. A 54, 225 (1975).

${ }^{27}$ B. A. Ravan, A. A. Shokri, and A. Yazdani, J. Appl. Phys. 109, 07C734 (2011).

${ }^{28}$ Y.-M. Chang, K.-S. Li, H. Huang, M.-J. Tung, S.-Y. Tong, and M.-T. Lin, J. Appl. Phys. 107, 093904 (2010). 